\title{
Karyotypic Evolution and Molecular Cytogenetic Analysis of Solanum pinnatisectum, a New Source of Resistance to Late Blight and Colorado Potato Beetle in Potato
}

\author{
Hai Yan Li*, Qin Chen, Debbie Beasley, Dermot R. Lynch and Mark Goettel \\ Agriculture and Agri-Food Canada, Lethbridge Research Centre, Lethbridge, AB T1J 4B1, Canada
}

Received October 31, 2005; accepted December 22, 2005

\begin{abstract}
Summary To efficiently transfer important disease and insect resistance traits from diploid Mexican wild species Solanum pinnatisectum to tetraploid cultivated Solanum tuberosum, molecular cytogenetic and karyotypic analyses were carried out to examine the degree of chromosomal and genomic variation between these 2 species. The results demonstrated that the chromosome complement in S. pinnatisectum was predominantly metacentric and submetacentric, while S. tuberosum showed prominent subtelocentric and telocentric chromosomes. It appears that karyotype evolution from diploid to tetraploid originates through chromosome rearrangements, involving mainly deletions or multiplication at the same ploidy level. Fluorescence in situ hybridization (FISH) using 18S-5.8S26S ribosomal RNA (rDNA) as a probe demonstrated that both diploid and tetraploid potato species had a single nucleolus organizer region (NOR) locus. However, the localization of the rDNA sites in one of the 2 accessions of $S$. pinnatisectum was characterized by a pair of morphologically distinct chromosomes, in which one was a submetacentric chromosome, while its homologous partner was a subtelocentric chromosome, which was very similar to that observed in tetraploid potato. This indicated that chromosome variation in structural alterations and nuclear DNA content, as well as loss or addition of highly repetitive sequences could play a role in potato evolution and development of new cultivars. This study offers a useful molecular cytogenetic marker for species identification, chromosome inheritance and potential introgression in future intergenetic hybridization experiments with Mexican wild potato species.
\end{abstract}

Key words Chromosome organization, Evolution, FISH, Mexican wild species, S. tuberosum, 18S5.8S-26S rRNA.

Potato (Solanum tuberosum L.) is one of the most important horticultural and food crops in the world. However, disease and insect damage caused by late blight [Phytophthora infestans (Mont.) de Bary] and Colorado potato beetle (CPB, Leptinotarsa decemlineata Say) have a great effect on the production of potato (Fry and Goodwin 1997). The development of new potato cultivars with high levels of genetic resistance to late blight and CPB would significantly improve potato production. Previous studies have identified several wild species, especially a Mexican wild diploid species Solanum pinnatisectum, possess high levels of genetic resistance to both late blight and CPB (Douches et al. 2001, Chen et al. 2003a). The valuable Mexican wild species could be used as a potential gene pool to improve potato for disease and insect resistance. However, it is very difficult to transfer desirable traits from $S$. pinnatisectum to $S$. tuberosum because of sexual incompatibility barriers. A better understanding of the genetic relationship and genomic closeness between these 2 species may greatly assist breeders in successfully transferring useful genes from the wild species into cultivated potato.

Plant cytogenetic and genomic analyses have provided useful information about chromosome

* Corresponding author, e-mail: lihai@agr.gc.ca 
structure and behavior that are necessary to understand the fundamental processes in plant cells, which helps plant breeders with the introduction of genes from related or more distant species (Sybenga 1992). Molecular cytogenetic techniques, such as fluorescence in situ hybridization (FISH) are valuable methods to characterize whole chromosomes or chromosome segments, which could give a complementary view for various levels of chromosomal evolution and relationships of species, and help to advance the understanding of the genetic basis of natural variation for the cultivated and wild potato species (Chen et al. 1998, Dong et al. 2000). Comparison of karyotype and nuclear DNA in diploid and tetraploid potato species provides valuable information related to the mechanisms of genome evolution in cytotaxonomic and evolutionary studies. However, chromosome identification is more of a challenge in many plant species with small chromosomes, such as potato. Although some karyotype descriptions have been reported in potato (Yeh and Peloquin 1965, Visser et al. 1988, Mohanty et al. 2004), cytogenetical information still lack for both diploid and tetraploid in the genus Solanum, especially for the diploid Mexican wild species (Wilkinson 1994). This is mainly due to problems associated with difficulties in the preparation of good chromosome spreads, small chromosome size with very similar morphology, and the low natural frequency of synchronously dividing cells in root-tip meristems (Hawkes 1979). Recently, a high quality, well-spread chromosome preparation procedure using Ambush as pretreatment agency has been developed in our laboratory (Chen and $\mathrm{Li}$ 2005), which enables routine work on chromosome preparation, karyotyping analysis, and molecular cytogenetic studies in potato.

In this study, the improved chromosome preparation procedure was used to produce wellspread metaphases for chromosome morphologic and karyotyping analysis in diploid Mexican species $S$. pinnatisectum and tetraploid cultivated S. tuberosum. FISH technique was applied to the physical mapping of potato genomes using the genes encoding $18 \mathrm{~S}-5.8 \mathrm{~S}-26 \mathrm{~S}$ ribosomal DNA as a probe. In addition, the karyotypes of these species were compared with their morphology, crossability, and DNA markers for the assessment and examination of chromosomal organization and degree of chromosomal variation among the Mexican wild and cultivated potato species.

\section{Material and methods}

\section{Plant materials and chromosome preparation}

Potato materials used in this study included 30 plants from 2 accessions PI 275233 and PI 275236 of a Mexican wild species $S$. pinnatisectum $(S$. pnt, $2 n=2 x=24)$ and 10 plants from one breeding line V0123-25 of the cultivated potato S. tuberosum $(S . t b r, 2 n=4 x=48)$. For chromosome preparation, root tips from 4-week old plants were pretreated with 1-10 ppm Ambush (a synthetic pyrethroid insecticide, ICI America, Inc., Wilmington, DE) at $4{ }^{\circ} \mathrm{C}$ for $20 \mathrm{~h}$. After pretreatment, the fixation of root tips, making chromosome slides and chromosome observation closely followed our previous methods (Chen and Li 2005). At least 5 metaphases of each root tip and 100-150 metaphase cells for each line were counted for chromosome numbers.

\section{Karyotypic analysis}

The chromosomes obtained from Ambush pretreatment were used for karyotypic analysis based on chromosome morphology. The metaphase chromosomes from 4 or more well-spread root tip cells per line were measured to obtain the average chromosome lengths and arm ratios. In all karyotypes, the chromosomes were classified according to their arm ratio (long/short) as metacentric (m: less than 1.19), submetacentric (sm: 1.20-1.99), subtelocentric (st: 2.00-3.99), and telocentric (t: above 4.00) (Kutarekar and Wanjari 1983, Gill et al. 1991). The twelve chromosome pairs in each genome were designated with numbers 1-12, which were arranged in order of decreasing total length. The chromosome length, position of centromere, and presence of secondary constrictions were taken into account in the karyotypic analysis (Swaminathan 1954). 
Table 1. Measurements of the length and arm ratio of chromosomes present in S. pinnatisectum (S. pnt, $2 n=2 x=24)$ and $S$. tuberosum (S. tbr, $2 n=4 x=48)$

\begin{tabular}{|c|c|c|c|c|c|c|}
\hline Lines & $\begin{array}{c}\text { Chromosome } \\
\text { no. }\end{array}$ & $\begin{array}{l}\text { Long arm } \\
\qquad(\mu \mathrm{m})\end{array}$ & $\begin{array}{l}\text { Short arm } \\
\qquad(\mu \mathrm{m})\end{array}$ & $\begin{array}{l}\text { Total } \\
(\mathrm{mm})\end{array}$ & $\begin{array}{c}\text { Arm ratio } \\
(\mathrm{L} / \mathrm{S})\end{array}$ & $\begin{array}{c}\text { Chromosome } \\
\text { type }^{\mathrm{b}}\end{array}$ \\
\hline S. pnt & $1^{*}$ & 4.98 & 2.65 & 7.63 & 1.88 & $\mathrm{sm}$ \\
\hline \multirow[t]{13}{*}{ PI 275233} & $1-1^{\mathrm{a}}$ & 5.26 & 2.90 & 8.16 & 1.81 & $\mathrm{sm}$ \\
\hline & $1-2$ & 4.70 & 2.39 & 7.09 & 1.97 & $\mathrm{sm}$ \\
\hline & 2 & 4.64 & 2.33 & 6.97 & 1.99 & $\mathrm{sm}$ \\
\hline & 3 & 3.85 & 1.92 & 5.77 & 2.01 & st \\
\hline & 4 & 2.88 & 2.08 & 4.96 & 1.39 & $\mathrm{sm}$ \\
\hline & 5 & 2.55 & 2.05 & 4.65 & 1.24 & $\mathrm{sm}$ \\
\hline & 6 & 2.40 & 2.10 & 4.50 & 1.14 & $\mathrm{~m}$ \\
\hline & 7 & 2.50 & 1.58 & 4.08 & 1.58 & $\mathrm{sm}$ \\
\hline & 8 & 2.13 & 1.88 & 4.01 & 1.13 & $\mathrm{~m}$ \\
\hline & 9 & 2.50 & 1.38 & 3.88 & 1.82 & $\mathrm{sm}$ \\
\hline & 10 & 2.20 & 1.63 & 3.83 & 1.35 & $\mathrm{sm}$ \\
\hline & 11 & 1.88 & 1.85 & 3.73 & 1.02 & $\mathrm{~m}$ \\
\hline & 12 & 1.78 & 1.31 & 3.09 & 1.36 & $\mathrm{sm}$ \\
\hline S. pnt & $1^{*}$ & 5.79 & 3.39 & 9.18 & 1.71 & $\mathrm{sm}$ \\
\hline \multirow[t]{13}{*}{ PI 275236} & $1-1^{a}$ & 7.37 & 5.26 & 12.63 & 1.40 & $\mathrm{sm}$ \\
\hline & $1-2$ & 4.21 & 1.52 & 5.73 & 2.77 & st \\
\hline & 2 & 4.83 & 3.10 & 7.93 & 1.56 & $\mathrm{sm}$ \\
\hline & 3 & 3.69 & 3.25 & 6.95 & 1.14 & $\mathrm{~m}$ \\
\hline & 4 & 4.14 & 2.06 & 6.20 & 2.01 & st \\
\hline & 5 & 3.06 & 2.29 & 5.35 & 1.34 & $\mathrm{sm}$ \\
\hline & 6 & 2.74 & 2.17 & 4.91 & 1.26 & $\mathrm{sm}$ \\
\hline & 7 & 4.17 & 0.57 & 4.74 & 7.30 & $\mathrm{t}$ \\
\hline & 8 & 2.86 & 1.71 & 4.57 & 1.67 & $\mathrm{sm}$ \\
\hline & 9 & 2.57 & 1.89 & 4.46 & 1.36 & $\mathrm{sm}$ \\
\hline & 10 & 2.76 & 1.35 & 4.11 & 2.04 & st \\
\hline & 11 & 2.00 & 2.00 & 4.00 & 1.00 & $\mathrm{~m}$ \\
\hline & 12 & 1.83 & 1.37 & 3.20 & 1.33 & $\mathrm{sm}$ \\
\hline S. $t b r$ & 1 & 3.93 & 1.92 & 5.85 & 2.05 & st \\
\hline \multirow[t]{11}{*}{ V0123-25 } & 2 & 2.44 & 2.17 & 4.61 & 1.13 & $\mathrm{~m}$ \\
\hline & $3^{*}$ & 3.53 & 1.17 & 4.70 & 3.02 & st \\
\hline & 4 & 2.57 & 1.74 & 4.31 & 1.48 & $\mathrm{sm}$ \\
\hline & 5 & 2.33 & 1.94 & 4.27 & 1.20 & $\mathrm{sm}$ \\
\hline & 6 & 2.29 & 1.74 & 4.03 & 1.32 & $\mathrm{sm}$ \\
\hline & 7 & 2.64 & 1.32 & 3.96 & 2.00 & st \\
\hline & 8 & 2.28 & 1.67 & 3.95 & 1.37 & $\mathrm{sm}$ \\
\hline & 9 & 2.85 & 0.63 & 3.48 & 4.56 & $\mathrm{t}$ \\
\hline & 10 & 1.94 & 1.25 & 3.19 & 1.56 & $\mathrm{sm}$ \\
\hline & 11 & 1.54 & 1.52 & 3.06 & 1.01 & $\mathrm{~m}$ \\
\hline & 12 & 2.50 & 0.28 & 2.78 & 9.00 & $\mathrm{t}$ \\
\hline
\end{tabular}

* FISH signal chromosomes. ${ }^{\mathrm{a}}$ One of the two homologous chromosomes. ${ }^{\mathrm{b}} \mathrm{m}, \mathrm{sm}$, st, and $\mathrm{t}$ represent metacentric, submetacentric, subtelocentric, and telocentric, respectively.

FISH

Clone pTa71, which was kindly provided by Dr. B. S. Gill, Kansas State University, Manhattan, USA, was used as a probe for a cytogenetic marker. It is a 9-kb EcoRI fragment of the 18S5.8S-26S ribosomal RNA (rDNA) genes and intergenic spacers isolated from Triticum aestivum recloned into pUC19. The entire plasmid was labeled with biotin-14-dUTP by nick translation according to the manufacturer's recommendations. About $3 \mathrm{mg}$ of DNA were labeled per $50 \mu \mathrm{l}$ of labeling reaction (using $5 \mathrm{ml}$ of $10 \times$ enzyme mix) for $2 \mathrm{~h}$ at $16^{\circ} \mathrm{C}$, and the reaction was stopped with $5 \mu \mathrm{l}$ of $300 \mathrm{mM}$ EDTA ( $\mathrm{pH} 8.0$ ) solution. Unincorporated nucleotides were removed by passing the labeling reaction through a Sephadex G-50 column (Millipore, Bedford, MA). The in situ hy- 
A

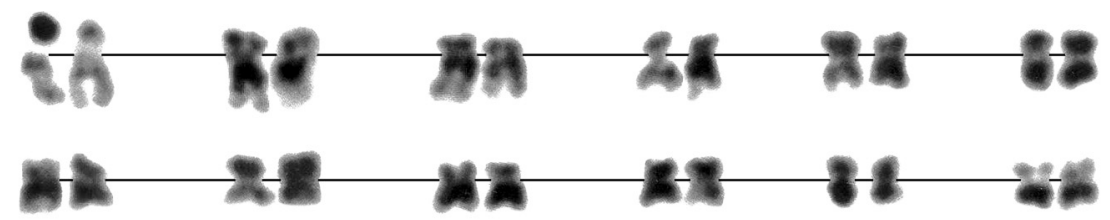

B

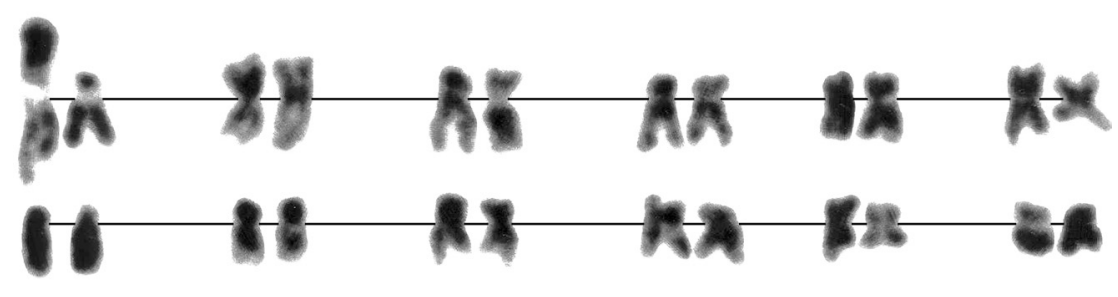

C

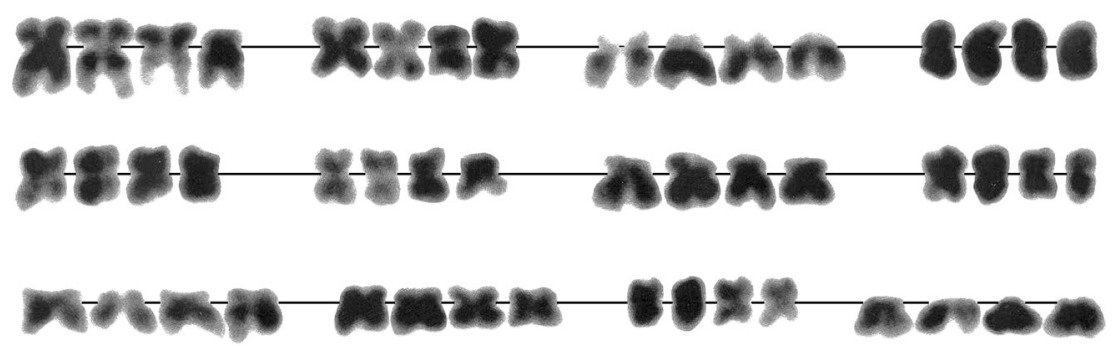

Fig. 1. Karyotypic analysis. A) Mexican diploid species S. pinnatisectum PI 275233. B) Mexican diploid species S. pinnatisectum PI 275236. C) Tetraploid potato line V0123-25.

bridization procedure described by Chen et al. (1998) was adopted with a minor modification of denaturing the chromosome slides with $70 \%$ Formamide in $2 \times \mathrm{SSC}$ for $1.5 \mathrm{~min}$ at $80^{\circ} \mathrm{C}$. Slides were examined using an Olympus BX51 epifluorescence microscope with red and green filter sets. Photographs were taken and processed with SPOT imaging software (Diagnostic Instruments, Inc.)

\section{Results}

Cytogenetic examination showed that the 2 accessions of the diploid Mexican species S. pinnatisectum had 24 chromosomes. However, absence of 1 to 2 chromosomes in some root tip cells was observed in different plants at a very low frequency $(\sim 5 \%)$. All of the 10 plants tested in the cultivated potato $S$. tuberosum had 48 chromosomes, as expected for a tetraploid potato species. The chromosomes obtained from Ambush pretreatments had clear centromeres for all chromosomes in the 2 species, which facilitated the study of chromosomal and structural variations in genomes. We chose well-spread metaphases in the diploid and tetraploid species for karyotyping observation and FISH analysis.

\section{Accession PI 275233 of the Mexican diploid species S. pinnatisectum}

The lengths of each of the 12 pairs of chromosomes in PI 275233 were quite similar, ranging from 3.09 to $7.63 \mu \mathrm{m}$. In some cells, the highly contracted metaphase chromosomes were approximately half of their normal lengths. Its genome karyotype was composed of 3 pairs of metacentric (chromosomes 6, 8, and 11), 8 pairs of submetacentric (chromosomes 1, 2, 4, 5, 7, 9, 10, and 12), and 1 pair of subtelocentric chromosomes (chromosome 3) (Table 1). Constriction-like regions were constantly observed near the central area of the long arms of the chromosomes 1, 2 and 3 that 

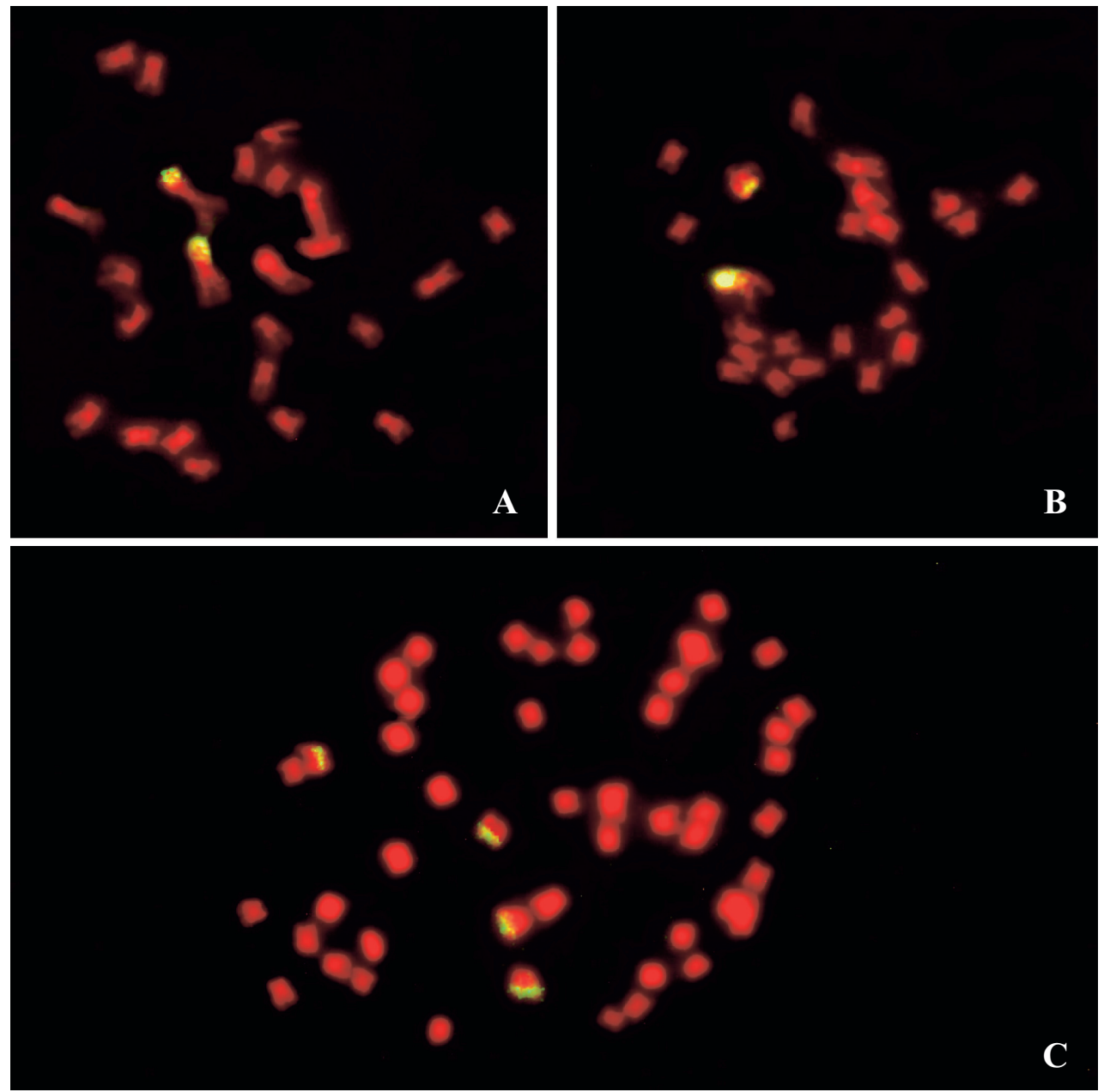

Fig. 2. Chromosomal assignments of $18 \mathrm{~S}-5.8 \mathrm{~S}-26 \mathrm{~S}$ rRNA gene by FISH. A) Two hybridization signals on chromosome 1 in Mexican diploid species S. pinnatisectum PI 275233. B) Two signals on chromosome 1 in $S$. pinnatisectum PI 275236. C) Four signals on chromosome 3 in the tetraploid potato line V0123-25.

aided their identifications. The nearly equal arm length was a distinguishing characteristic of these 3 chromosomes that set them apart from the other medium-sized ones. Satellite chromosomes were occasionally found separately or attached to the short arm of chromosome 1 in about $20 \%$ of cells observed (data not shown). The lengths of the 2 short arms of chromosome 1 were similar, at 2.39 and $2.90 \mu \mathrm{m}$ with an arm ratio of 1.97 and 1.81, respectively. Chromosome 12 was the shortest submetacentric chromosome. Its size and symmetrical appearance made it easy to be recognized (Fig. 1A).

FISH on chromosome spreads of accession PI 275233 with pTa71 probe displayed 2 clear hybridization signals on the short arms of each of the homologous chromosome 1 (Fig. 2A). These 2 sites showed similar signal dispersion, although minor size difference of the hybridization signal loci was noted on the 2 arms of the homologous chromosomes during their genotype analysis. Those constriction-like regions in the long arms of chromosomes 1, 2 and 3 did not show rDNA signals. 


\section{Accession PI 275236 of the Mexican species S. pinnatisectum}

The total lengths in accession PI 275236 were ranged from 3.20 to $9.18 \mu \mathrm{m}$. Its karyotype was somehow different from that of the accession PI 275233 (Fig. 1B). PI 275236 carried 2 pairs of metacentric (chromosomes 3 and 11), 7 pairs of submetacentric (chromosomes 1, 2, 5, 6, 8, 9, and 12), 2 pairs of subtelocentric (chromosomes 4 and 10), and 1 pair of telocentric chromosomes (chromosome 7). It was noted that there was a very long chromosome in all cells observed in this accession. Since nucleolus organizer region (NOR) was always attached to the short arm of the chromosome, this made the chromosome the longest one with a total length of $12.63 \mu \mathrm{m}$. Its short arm was $5.26 \mu \mathrm{m}$ with an arm ratio of 1.40 . However, its homologous chromosome was a subtelocentric chromosome, its short arm was only $1.52 \mu \mathrm{m}$ with an arm ratio of 2.77 (Table 1). A pair of chromosomes including 1 the longest submetacentric chromosome plus 1 subtelocentric chromosome was the characteristic of karyotype for the accession PI 275236. Constriction-like regions were observed in chromosomes 1, 2, 3 and 4 on their long arms.

Two rDNA loci were also seen on each of the homologous chromosome 1 in PI 275236. It was clear that the size of the hybridization signals was different: one pronounced signal occurred along almost the whole short arm of the largest submetacentric chromosome 1, while another minor signal was located only at the end of the short arm of its homologous subtelocentric chromosome (Fig. 2B). Constriction-like regions didn't show any signals in this accession.

\section{V0123-25 of the cultivated tetraploid S. tuberosum}

The total length for each chromosome in the tetraploid species $S$. tuberosum ranged from 2.78 to $5.85 \mu \mathrm{m}$ (Table 1). Its genome was composed of 2 pairs of metacentric (chromosomes 2 and 11), 5 pairs of submetacentric (chromosomes 4, 5, 6, 8, and 10), 3 pairs of subtelocentric chromosomes (chromosomes 1, 3 and 7), and 2 pairs of telocentric chromosomes (chromosomes 9 and 12). But compared with the diploid species, constriction-like areas were only found on the long arms of chromosome 1. Satellite chromosomes were not observed in this species (Fig. 1C).

The pTa71 probe revealed 4 hybridization signals at the distal end of the short arm of chromosome 3 and the signal strength was approximately equal among the 4 telocentric chromosomes at all metaphases in the tetraploid cultivated line (Fig. 2C).

\section{Discussion}

The present results showed that chromosomes derived from Ambush pretreatment, which was previously proven to produce more distinguishable chromosomes (Chen and Li 2005), could be used for chromosome morphology, karyotypic, and molecular cytogenetic analyses in potato. Identification of the potato chromosomes based on their length, centromeric position, arm ratio, and morphological characteristics is possible at mitosis using the present technique. There has been no detailed karyotype studies published for diploid Mexican species S. pinnatisectum, although some preliminary karyotype of dihaploid and tetraploid cultivated potato had been analysed (Yeh and Peloquin 1965, Visser et al. 1988, Mohanty et al. 2004). Using pachytene analysis of haploids, Yeh and Peloquin (1965) found no gross structural differences between haploids of the groups Tuberosum and Andigena. However, recent karyotypic analyses and studies on the nuclear DNA content showed that highly significant variations were observed in the genome length, volume and total form percentage at the cultivar level in 30 genotypes of cultivated potato (Mohanty et al. 2004). In the present study, karyotypic analysis of diploid Mexican S. pinnatisectum and tetraploid S. tuberosum for the number of metacentric and telocentric chromosomes demonstrated that, although most of the chromosomes were morphologically similar, there were large structural differences between these 2 species. The genome of $S$. pinnatisectum was mostly composed of metacentric and submetacentric chromosomes, while more subtelocentric or telocentric chromosomes were found in the $S$. 
tuberosum species. This indicated that structural rearrangements, such as deletions and translocations had occurred during the evolution of potato from diploid to tetraploid species. The reduction of the number of metacentric and constriction-like chromosomes might have occurred during the course of the evolution through mutations, and structural and physiological changes (Schultz-Schaeffer 1960, Mohanty et al. 2004). The longest chromosome 1 in both diploid and tetraploid species had constriction-like regions on the long arms. The constrictions were also observed in chromosomes 2 and 3 in PI 275233 and chromosomes 2, 3 and 4 in PI 275236. However, FISH did not show rDNA sites in all of these constriction chromosomes. Satellites were present only in some cells of one accession of diploid Mexican species tested, but were not observed in the tetraploid potato in the present study.

Chromosome differentiation not only exists between species, but also among accessions and genotypes in the Mexican S. pinnatisectum species. For example, the karyotypes of the two accessions of $S$. pinnatisectum showed that although they belonged to the same species, the number of metacentric, subtelocentric, and telocentric chromosomes were different. There were more metacentric and less subtelocentric or telocentric chromosomes in PI 275233 than in PI 275236 (Table 1). Variation was also observed in the same cell for the same pair of chromosomes, especially the homologous chromosomes of chromosome 1 in the Mexican species was morphologically distinct. These variations sometimes made it difficult to distinguish the same group or similar chromosomes on the basis of total length in the diploid species. The primary cause of the chromosome polymorphism in the somatic cells remains unknown at the present time. It could be due to the highly heterozygous and open-pollinated nature of potato.

The present study also demonstrated that the chromosomes obtained from Ambush pretreatments were suitable for FISH karyotypic analysis and physical mapping of genes or DNA sequences on chromosomes. FISH with pTa71 probe revealed different numbers of hybridization sites in the diploid and tetraploid species, and even different morphology, intensity and size of signals within the same group of chromosomes in the Mexican species. Since we had previously observed bivalent and quadrivalent pairings at meiosis in the two accessions of the diploid Mexican species and tetraploid potato species (unpublished data), the presence of the 2 or 4 hybridization sites was indicative of a single NOR rDNA locus on a set of 2 or 4 homologous chromosomes in the diploid and tetraploid species. As FISH is a semi-quantitative technique, the difference observed in the strength, intensity and the size of an observed fluorescent signal may reflect a variation in intergenic spacer size or in the number of repeat units. The weakly labeled chromosomes may possess a lower number of repeat units of the rDNA than the chromosomes indicating stronger signals (Harding and Millam 1999). The reason for the different size of 2 rDNA loci observed in a pair of homologous chromosome 1 in PI 275236 is presumed to be the result of one of the chromosomes undergoing a deletion during the course of evolution. The deletion within one of the homologous chromosomes was recognized also in other Solanum species (Srebniak et al. 2002, Mohanty et al. 2004). The differences observed for the rDNA probes and the expression of rDNA genes may reflect important differences in the chromosomal organization of repetitive DNA sequences among chromosomes between these 2 species. This structural variation could be caused by changes in DNA methylation pattern or loss of rDNA copies during the evaluation procedure (Chen and Pikaard 1997). The variation and structural rearrangements including loss or addition of highly repetitive sequences in the genome might provide an opportunity for karyotype evaluation and play an important role in developing new species.

It is interesting to note that rDNA signals in the deletion chromosomes in the diploid accession PI 275236 are very similar to the signal chromosomes observed in tetraploid potato (Fig. 2B, C). The karyotypic characterization of the PI 275236 was also similar to that of the tetraploid species (Table 1). Previous studies for crossability and disease resistance in Mexican species S. pinnatisectum showed that the diploid accession PI 275236 was more easily crossed with other species than 
the diploid accession PI 275233 (Chen et al. 2003b). The present study identified that PI 275236 had a closer karyotype and rDNA signal pattern with the tetraploid species $S$. tuberosome than PI 275233. The similarity of karyotype between PI 275236 and the cultivated potato would explain the genetic and crossability difference among the 2 Mexican species. The big signals observed in the short arm of chromosome 1 in the 2 accessions of the $S$. pinnatisectum species may be linked with high levels of disease and insect resistance identified in the Mexican diploid species. This specific chromosome could serve as a molecular cytogenetic marker for species identification and potential introgression in future intergenetic hybridization experiments with Mexican wild species. Thus the procedure presented in this study provides a useful means for detecting and characterizing structural rearrangements of chromosomes, which is useful for investigating phylogenetic relationships among the different potato species.

\section{Acknowledgements}

The financial supports provided by the Matching Investment Initiative of Agriculture and AgriFood Canada, the Potato Growers of Alberta, Midwest Food Products Inc., and Keystone Vegetable Producers Association Inc. are greatly acknowledged.

\section{References}

Chen, Q., Friebe, B., Conner, R. L., Laroche, A., Thomas, J. B. and Gill, B. S. 1998. Molecular cytogenetic characterization of Thinopyrum intermedium derived wheat germplasm specifying resistance to wheat streak mosaic virus. Theor. Appl. Genet. 96: 1-7.

—, Kawchuk, L. M., Lynch, D. R., Goettel, M. S. and Fujimoto, D. K. 2003a. Identification of late blight, Colorado potato beetle, and blackleg resistance in 3 Mexican and 2 South American wild $2 x$ (1EBN) Solanum species. Am. J. Potato Res. 80: 9-19.

— and Li, H. Y. 2005. An improved technique for high resolution mitotic chromosomes studies in Solanum. Hort. Science 40: $54-56$.

—, Lynch, D. R., Platt, H. W., Li, H. Y., Shi, Y., Li H. J. and Beasley, D. 2003b. Interspecific crossability and cytogenetic analysis of sexual progenies of Mexican wild diploid 1EBN species Solanum pinnatisectum and S. cardiophyllum. Am. J. Potato Res. 81: 159-169.

Chen, Z. J. and Pikaard, C. S. 1997. Epigenetic silencing of RNA polymerase I transcription: a role for DNA methylation and histone modification in nucleolar dominance. Gene Dev. 11: 2124-2136.

Dong, F., Song, J., Naess, S. K., Helgeson, J. P., Gebhardt, C. and Jiang, J. 2000. Development and applications of a set of chromosomes-specific cytogenetic DNA markers in potato. Theor. Appl. Genet. 101: 1001-1007.

Douches, D. S., Inglis, D. A., Helgeson, J. P. and Brown, C. R. 2001. Partial resistance to Pytophthora infestans in 4 Solanum crosses. Am. J. Potato Res. 78: 9-17.

Fry, W. E. and Goodwin, S. B. 1997. Re-emergence of potato and tomato late blight in the United States. Plant Dis. 81: 1349-1357.

Gill, B. S., Friebe, B. and Endo, T. R. 1991. Standard karyotype and nomenclature system for description of chromosomes bands and structural aberration in wheat (Triticum aestivum). Genome 34: 830-839.

Harding, K. and Millam, S. 1999. Analysis of ribosomal RNA genes in somatic hybrids between wild and cultivated Solanum species. Mol. Breed. 5: 11-20.

Hawkes, J. G. 1979. The biology and taxonomy of Solanaceae. In: Hawkes, J. G., Lester, R. N. and Skelding, A. D. (eds.). Evolution and Polyploidy in Potato Species. Academic Press, London. pp. 637-645.

Kutarekar, D. R. and Wanjari, K. B. 1983. Karyomorphological studies in some of the varieties of Bengal gram (Cicer arietinum L.). Cytologia 48: 699-705.

Mohanty, I. C., Mahapatra, D., Mohanty, S. and Das, A. B. 2004. Karytype analyses and studies on the nuclear DNA content in 30 genotypes of potato (Solanum tuberosum) L. Cell Biol. Int. 28: 625-633.

Schultz-Schaeffer, J. 1960. Cyotological investigation in the genus Bromus. III. The cytotaxonomic significance of the satellite chromosomes. J. Hered. 51: 269-277.

Srebniak, M., Rasmussen, O. and Maluszynska, J. 2002. Cytogenetic analysis of an asymmetric potato hybrid. J. Appl. Genet. 43: 19-31.

Swaminathan, M. S. 1954. Nature of polyploidy in some 48-chromosomes species of the genus Solanum, section Tubero- 
sum. Genetics 39: 59-76.

Sybenga, J. 1992. Cytogenetics in plant breeding. Springer, Berlin/Heidelberg.

Visser, R. G., Hoekstra, R., Vander, L., Pijnacker, L. P., Witholt, B. and Feenstra, W. J. 1988. In situ hybridization to somatic metaphase chromosomes of potato. Theor. Appl. Genet. 76: 420-424.

Wilkinson, M. J. 1994. Genome evolution in potatoes. In: Potato genetics. CAB International, Wallingford, Oxon, UK. pp. 43-70.

Yeh, B. P. and Peloquin, S. J. 1965. Pachytene chromosomes of the potato (Solanum tuberosum, Group Andigena). Am. J. Bot. 52: 1014-1020. 